\title{
Assessment of wind heeling lever determined through CFD against the current naval stability standards
}

\author{
J N Alderton* BSc (Hons) \\ * QinetiQ, Gosport, UK \\ * Corresponding Author. Email: jnalderton@qinetiq.com
}

\begin{abstract}
Synopsis
Naval stability standards consider the impact of a number of different external factors, one of which is the effect of heeling caused by wind. With relatively large superstructures the wind heeling moment can be relatively significant but despite its potential impact, at present the calculation to determine the wind heeling moment is relatively simplistic.
\end{abstract}

With increasing fidelity within computational tools, in particular Computational Fluid Dynamics (CFD), it questions whether the current standards are still considered fit for purpose or whether a more time consuming but comprehensive analysis should be used.

This paper discusses work conducted by QinetiQ on behalf of the UK MoD, to explore this area. The work firstly benchmarks wind heeling moment derived by different CFD methods against existing model wind tunnel test results for a heeled patrol boat. The benchmarking compares the level of accuracy of the numerical tools and explores the impact of changing different parameters within the analysis.

Following the benchmarking at model scale, CFD is used to calculate the wind heeling force on two ships at full scale. The two selected ships represent very different types of hullform and ship particulars. The results from the CFD analysis are then compared to the results determined using current naval standard wind heeling criteria.

This paper discusses the different CFD methodology applied, the results from the benchmarking, the comparison between the CFD results and those determined by applying the current naval standard criteria and the implications on the applicability of a CFD analysis rather than the current criteria.

Keywords: Wind heeling; CFD; Aerodynamics; Naval stability standards

\section{Introduction}

The Naval Authority Group (NAG) is required by the Defence Maritime Regulator to set standards and independently assess compliance for the issue of safety certification to assure against capsize. This work includes the maintenance and upkeep of stability standards. The naval stability standards consider the impact of a number of different external factors, one of which is the effect of heeling caused by the wind.

The international Naval Stability Standards Working Group has been assessing the influence of wind forces on a ship's stability and has compared many approaches. Currently, the method of determining a ship's stability in beam winds is set out in DefStan 02-900 [1], where the naval standard for wind heeling criteria is defined.

Since 2010, the naval stability criterion, set out in DefStan 02-900, required the wind heeling moment to be calculated for an upright, intact ship which is then assumed to reduce with angle of heel $(\varnothing)$ as a function of $\operatorname{cosine}^{2} \varnothing$. This is due to the reduction in profile area and in the distance to the centre of lateral resistance. The preferred method for calculating the upright heeling moment is based on the work of Sarchin and Goldberg [2], where the ship's above water profile is split into layers from the waterline up. The profile area of each layer is then multiplied by the wind pressure for the corresponding layer, and then multiplied by the lever.

The calculations at present are seen to be relatively simplistic, accounting only for wind profile acting upon the ship profile from the waterline upwards. The effect of transient wake structures and recirculation on the leeward side of the ship is neglected. The use of Wind Tunnel (WT) testing addresses the effect of the wake, however there are practical limitations to WT testing, such as time and expense.

Authors' Biography

James Alderton is a Senior CFD Engineer within QinetiQ’s Analysis, Modelling \& Simulation CFD team. He has worked for QinetiQ since 2014 and has been involved predominantly in surface ship hydrodynamic and aerodynamic analysis. James' previous role was Senior Engineering Consultant for CD-adapco (now Siemens PLM), providing engineering support across a diverse range of industries from consumer products to oil and gas, but focusing predominantly in marine, automotive and motorsport. 
With significant improvements to High Powered Computing (HPC) and the reduction in computational hardware costs, the use of high fidelity computational tools such as CFD is becoming far more cost effective and readily available. However, the results of CFD calculations are only as reliable as their chosen computational models, with work needed to explore the accuracy of the models. Because of this, there is a requirement to benchmark the CFD processes with other prediction methods, such as WT testing.

In 2010, work was carried out by Khee [3] which detailed WT tests, conducted by the University of New South Wales (UNSW) for the Royal Australian Navy (RAN). The model used was a Patrol Boat (ACPB) at five heel angles, from upright through to 90 degrees. The work compared the empirical calculations of the wind heeling moment on the ship using the layered, strip method, with the measured WT experimental results. The work of Khee showed that the current DefStan approach matched well to the WT results for side force, with heeling moment being slightly under predicted. The WT results also showed that the wind heeling moment does not follow the assumption taken by the International Maritime Organisation (IMO), where it reduces to zero at high angles of heel.

In 2015, the work of Khee was taken further by Edwards [4], scaling up the WT data to full scale, then comparing the data with empirical calculations using the DefStan approach on a full scale profile.

This paper discusses the work carried out by QinetiQ, on behalf of the UK MoD, to benchmark the wind heeling moment derived from the CFD process, against the WT results carried out by UNSW and the empirical calculations using the DefStan approach. Following successful benchmarking of the CFD process, the same methodology was applied to the ACPB at full scale, comparing the results from CFD with scaled up WT results. Finally, the methodology was applied to a modern frigate geometry.

\section{Comparison of CFD with wind tunnel experimental data}

\subsection{Wind tunnel geometry}

The WT test was carried out on a simplified scale model of the RAN ACPB, consisting of the hull, superstructure, wheelhouse and mast, with secondary parts which would have little impact on the main projected area removed, such as antennas, ancillary craft and machinery. The 3D Computer Aided Design (CAD) model used to construct the WT model was imported into the CAD package Rhinoceros in the upright condition (Figure 1, left).
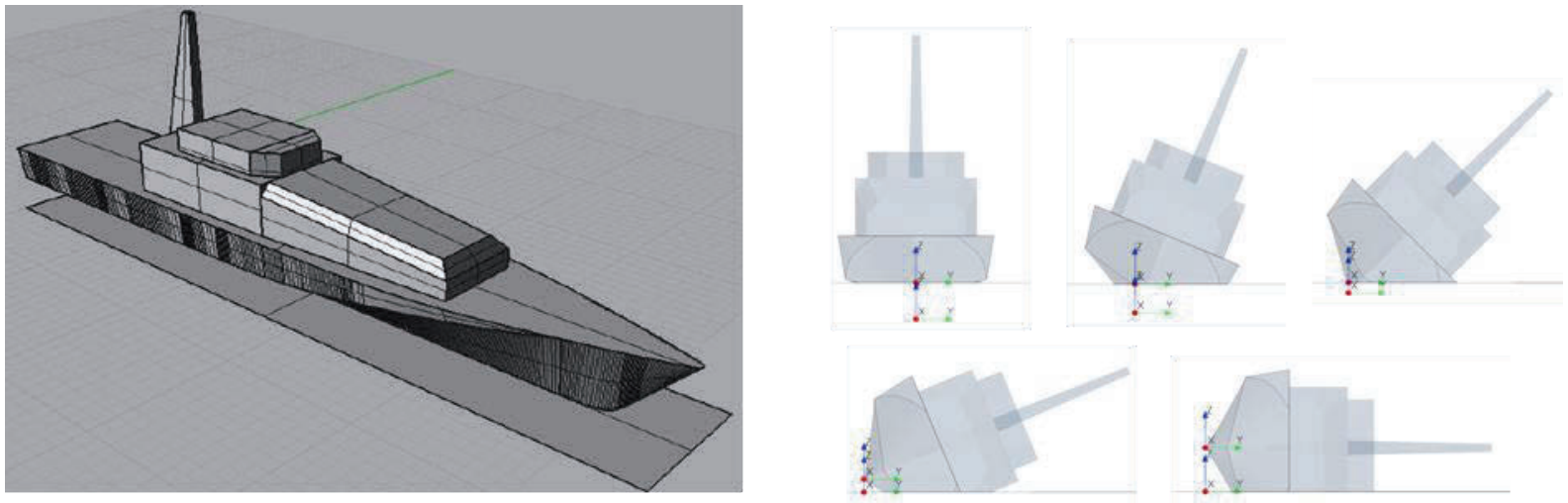

Figure 1: ACPB 3D CAD model at five different angles of heel

Khee [3] stated that the WT model was heeled to leeward about the keel line, with a base moulded around the model which replicated the waterline (WL), the draught being calculated assuming constant displacement and zero trim. Figure 1, right, illustrates the heeled geometries.

The CFD solver selected for this analysis was STAR-CCM+ V11.02, with the CFD WT domain created using the solvers built in 3D CAD modeller (Figure 2). The dimensions of the domain were taken from the report by Khee [3]. Images of the wind tunnel (Figure 3) show the tunnel edges were chamfered, so this was included in the CFD domain, resulting in a more representative model of the WT. The WT model of the ACPB was bolted to the corresponding angled base, and the base was then bolted to the Pyramidal Balance (PB) plate. 


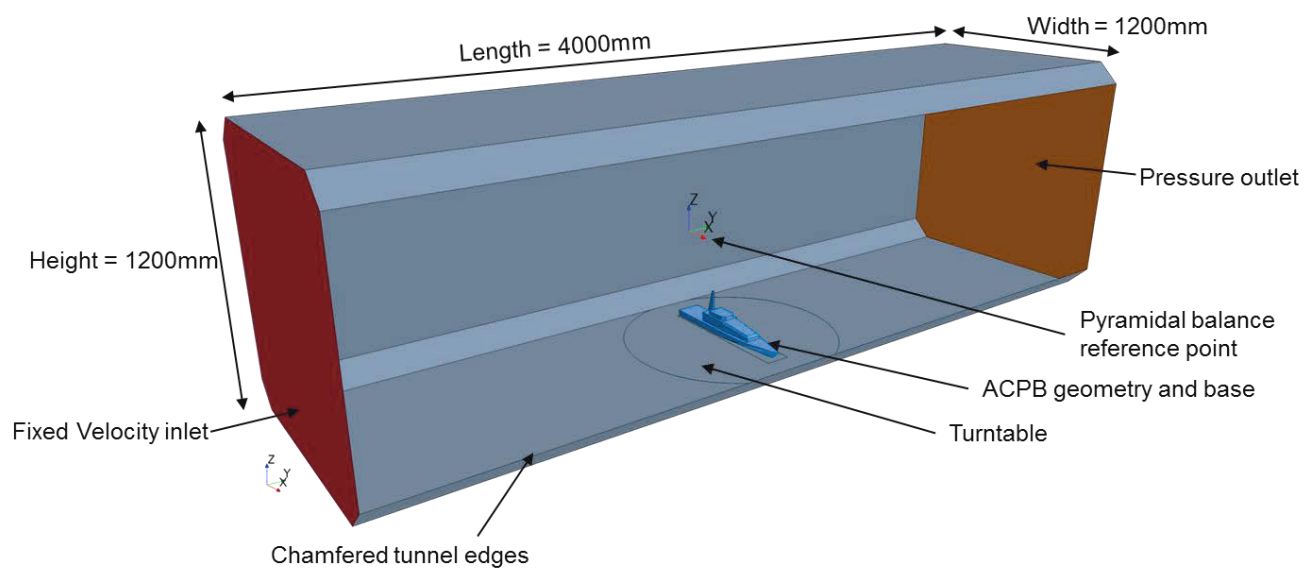

Figure 2: CFD WT domain

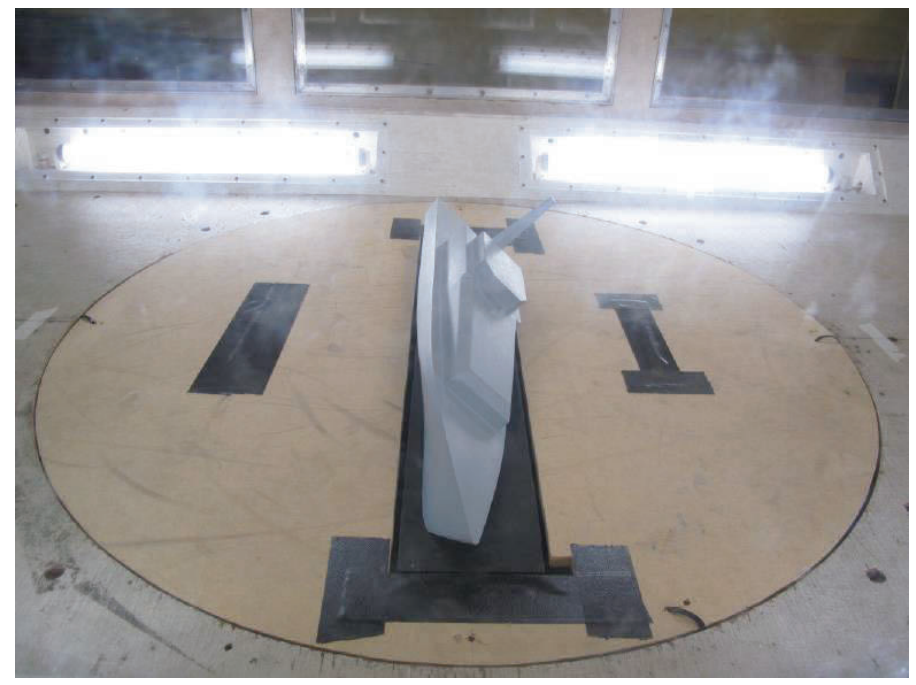

Figure 3: ACPB inside the UNSW wind tunnel test section, [3]

Forces and moments on the ACPB model were measured during the WT tests using the PB which was calibrated about a central reference point above the WT floor plane. The CFD model was set up to output force components on the ACPB as well as the moment about the PB reference point. This allowed for the CFD results to be directly compared with the raw wind tunnel data. The model was placed in the WT perpendicular to the flow, therefore the reported drag on the model was actually the side force on the ACPB.

\subsection{Simulation approach}

The flow calculations were carried out using STAR-CCM+ V11.02. The aim of this study was to recreate the WT test carried out by UNSW using CFD and to benchmark the CFD process using the raw WT test data. As the ACPB geometry was modelled within a controlled WT environment with a constant uniform air flow, the CFD simulations were set up to use a constant air density and assumed to be incompressible.

As the ACPB was placed at 90 degrees to the incoming flow, a large turbulent wake would be generated, which was considered important to the solution, therefore the CFD simulations were set up to compute the transient time dependant wake structures by solving for an implicit unsteady time solution.

The Shear Stress Transport (SST) K-Omega Unsteady Reynolds-Averaged Navier-Stokes (URANS) turbulence model was used. The preferred wall treatment used in the simulations was the all $\mathrm{y}+$ wall treatment model. This uses the wall $y+$ to determine whether the viscous sublayer is resolved (wall $y+<2$ ), or if a wall function approach is necessary (wall $\mathrm{y}+>30$ ).

In addition to running URANS, the SST K-Omega Detached-Eddy Simulation (DES) turbulence model was evaluated, which offers improved resolution of large eddy structures in the wake and is expected to be more accurate than a URANS based solution for unsteady wake flow. 
The ship was subjected to a constant wind speed of $40 \mathrm{~m} / \mathrm{s}$ from the inlet boundary, allowing the boundary layer to develop over the WT walls and floor which is a reasonable approximation of the true WT velocity profile. Forces and moments were reported and directly compared with the raw wind tunnel data.

As the results were transient, the solution was run until the transient fluctuations in the forces and moments had converged, with the results averaged over a sufficient number of time steps.

\subsection{Computational mesh}

The computational mesh setup used in the analysis had good mesh resolution within the wake region; an area of specific interest and relevant to this study. The boundary layer was adequately resolved using prismatic cells. The resulting wall y+ was less than one, ensuring the boundary layer was explicitly resolved.

As the intention was to assess the DES turbulence model, a mesh density was chosen which, when used in conjunction with a suitable time-step, resulted in sufficient resolution to achieve a convective courant number of one or less, allowing the eddies within the ship wake to be sufficiently resolved. Figure 4 shows the resulting upright 32 million trimmed hexahedral cell mesh which was used to carry out the WT scale analysis. The other four meshes varied by heel angle only and had similar cell counts.

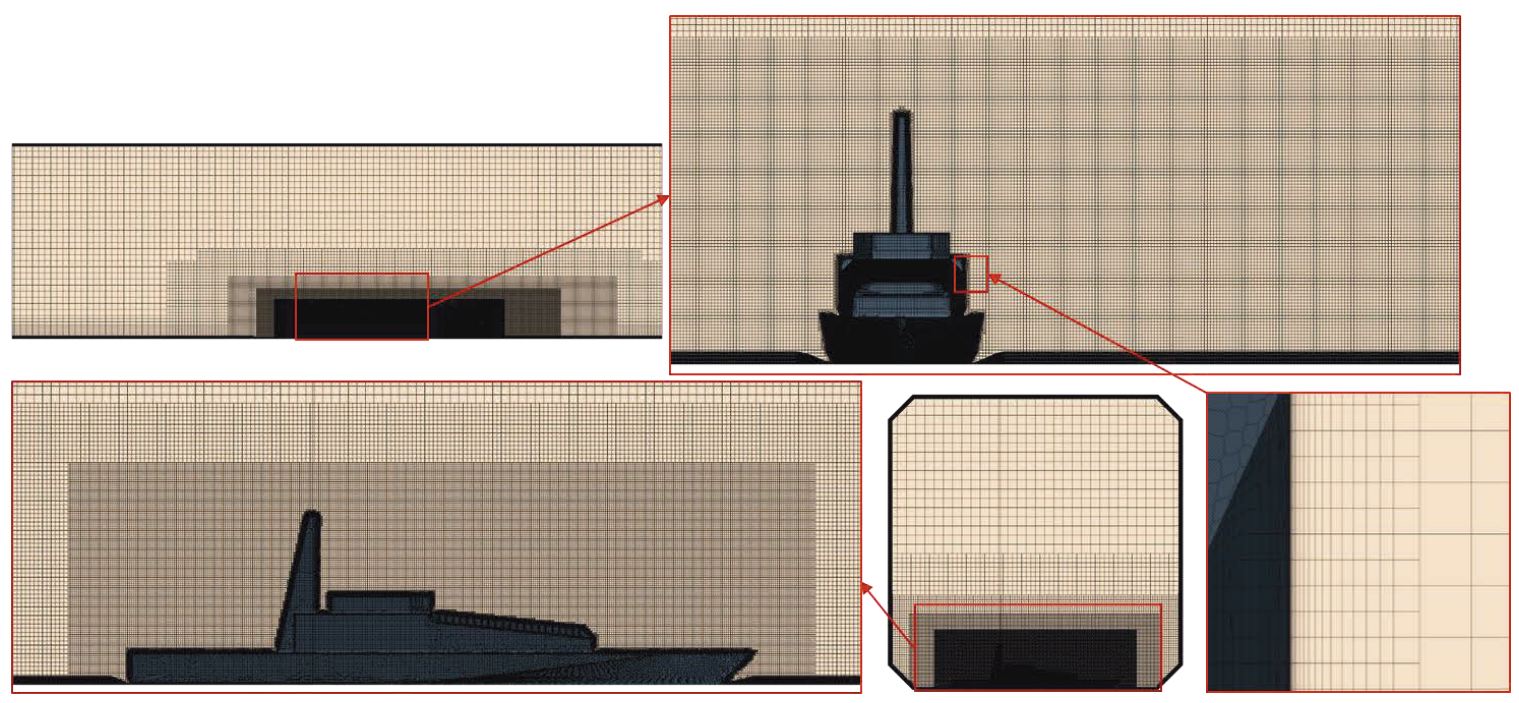

Figure 4: Computational WT ACPB mesh, approximately 32 million cells (upright)

\subsection{Wind tunnel scale turbulence model study}

The resulting computational meshes were used to conduct a turbulence model study, comparing the SST KOmega URANS model (henceforth referred to as SST) with the DES model. The results were compared directly with the raw ACPB WT data.

Figure 5 and Figure 6 show side force and heeling moment about the PB point for the model scale ACPB geometry at five heel angles. The results plotted are of the raw WT data (WT) and two CFD turbulence models.

In general the two turbulence models follow the same trend, varying only in magnitude. The SST results were found to follow the trend set by the WT test, yet consistently under-predicted. The DES results agreed well with those from the WT, predicting side force and heeling moment to within $4 \%$ of the WT test, with the exception of 90 degrees heel angle.

Resolving the turbulent eddies within the wake using DES overcomes the assumption of isotropic turbulence inherent in a two-equation turbulence model, and therefore gives a more accurate prediction of the size and strength of the recirculation, leading to a more accurate prediction of pressure and therefore side force. 


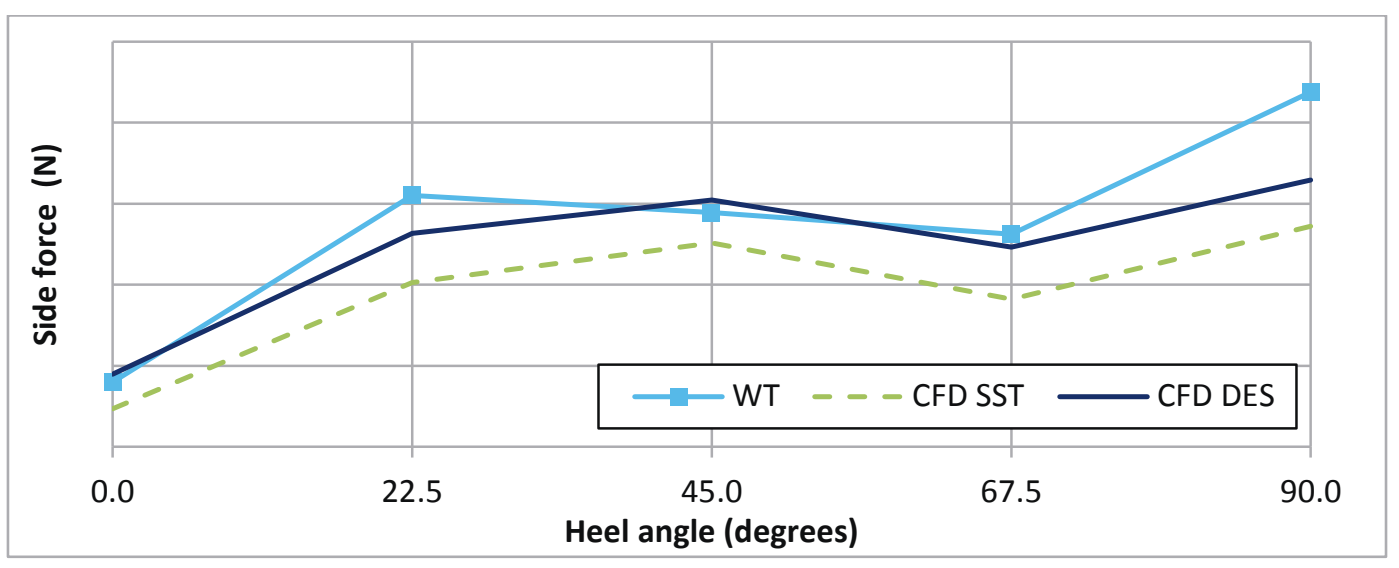

Figure 5: ACPB side force, $40 \mathrm{~m} / \mathrm{s}$

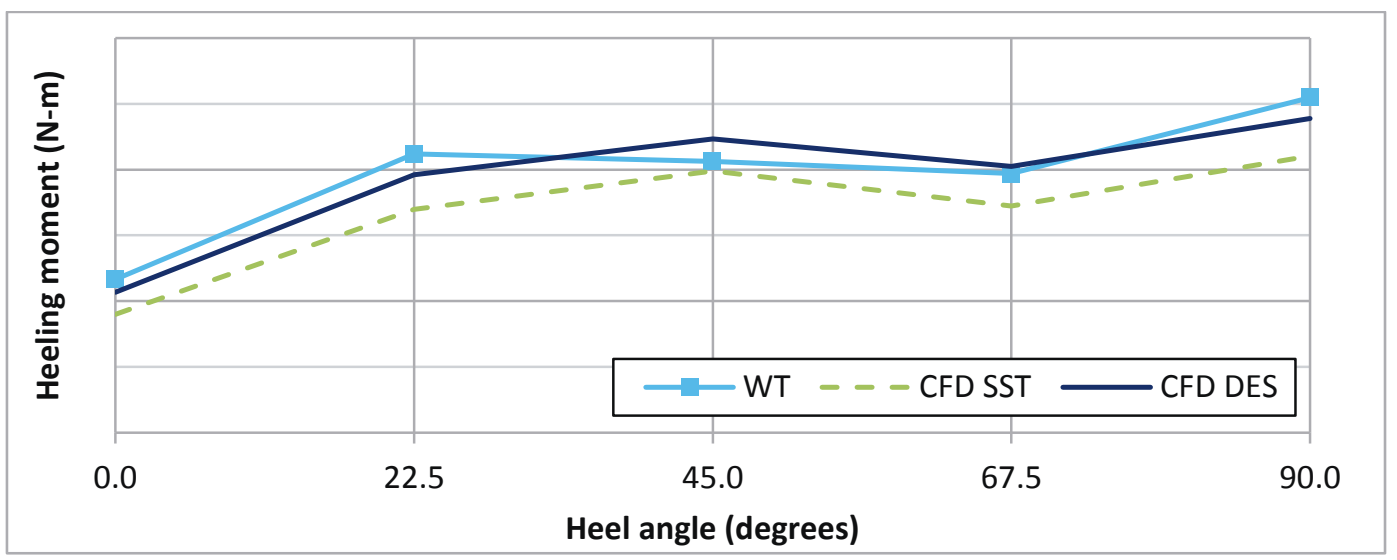

Figure 6: ACPB heeling moment, 40m/s

\subsection{Discussion of CFD WT results}

The turbulence model study showed that the DES model results agreed well with those from the WT. However, there were angles of heel where the predictions agreed better with the WT results than others. This could be attributed to geometric inaccuracies between what was modelled in the WT and what was modelled in CFD.

When looking at pictures of the ACPB model and bases used in the WT test, it was noted that at 22.5 degrees of heel, there was an additional section of the base removed to allow the ACPB model to be located (Figure 7). The removal of this section from the base increases the wetted surface area of the ACPB, potentially resulting in an increase in side force and heeling moment.

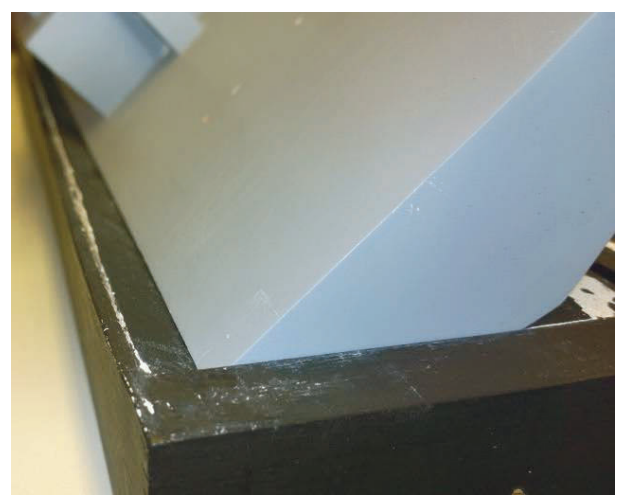

Figure 7: ACPB model and base at 22.5 degrees heel 
There are additional factors which could potentially contribute to the errors seen between the WT and CFD. These include the gaps between the base and the turntable, the turntable and the WT floor, even the small openings to access the fixing bolts. Although every effort would have been made to reduce these (with the use of tape for example, as seen in Figure 3) there is the potential for differences in the flow fields between the WT test and the CFD simulations. An example of this would be the development of a boundary layer prematurely breaking-up as it passes over the floor and turntable.

\section{Full scale predictions of the ACPB}

Following successful benchmarking of the ACPB geometry, the model scale WT CFD methodology was applied to the ACPB at full scale. This provided benchmarking against scaled up ACPB WT heeling force data and evaluated the methodology used in the CFD calculations to produce full scale wind heeling data.

\subsection{Full scale geometry}

For the full scale analysis, the same ACPB geometry was used. In the first instance, the WT walls were retained in the CFD model, such that the blockage was the same as at model scale. Subsequently, the domain geometry was modified to have open far-field boundaries further from the ship (Figure 8). The same constant wind speed of $40 \mathrm{~m} / \mathrm{s}$ was applied at the inlet boundary, allowing the boundary layer to develop over the WT walls and floor using the WT domain, and over the water surface of the far-field domain, which was modelled as a wall with a no-slip condition imposed. Both WT and open far-field domains were run and a comparison was made between the two, to determine the effects of blockage.

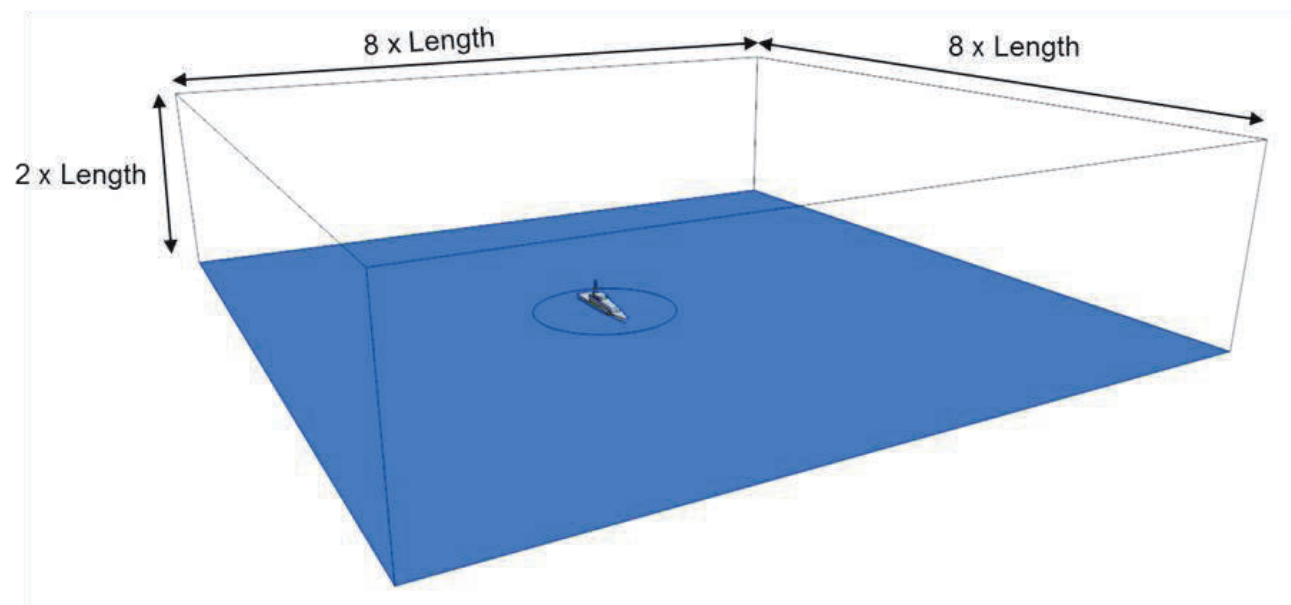

Figure 8: Open far-field full scale ACPB domain dimensions

\subsection{CFD domain influence at full scale}

In order to compare scaled up WT wind heeling forces with those from CFD, the full scale CFD simulations were run initially using a full scale WT domain, where the domain walls have an impact on the result. A second set of simulations were then run using a far-field domain. Figure 9 plots the side force on the ACPB at full scale, comparing scaled up WT wind heeling forces (WT) against full scale CFD wind heeling forces using the WT (CFD WT) domain, using the SST and DES turbulence models. Figure 10 plots the results using the farfield domain (CFD far-field). 


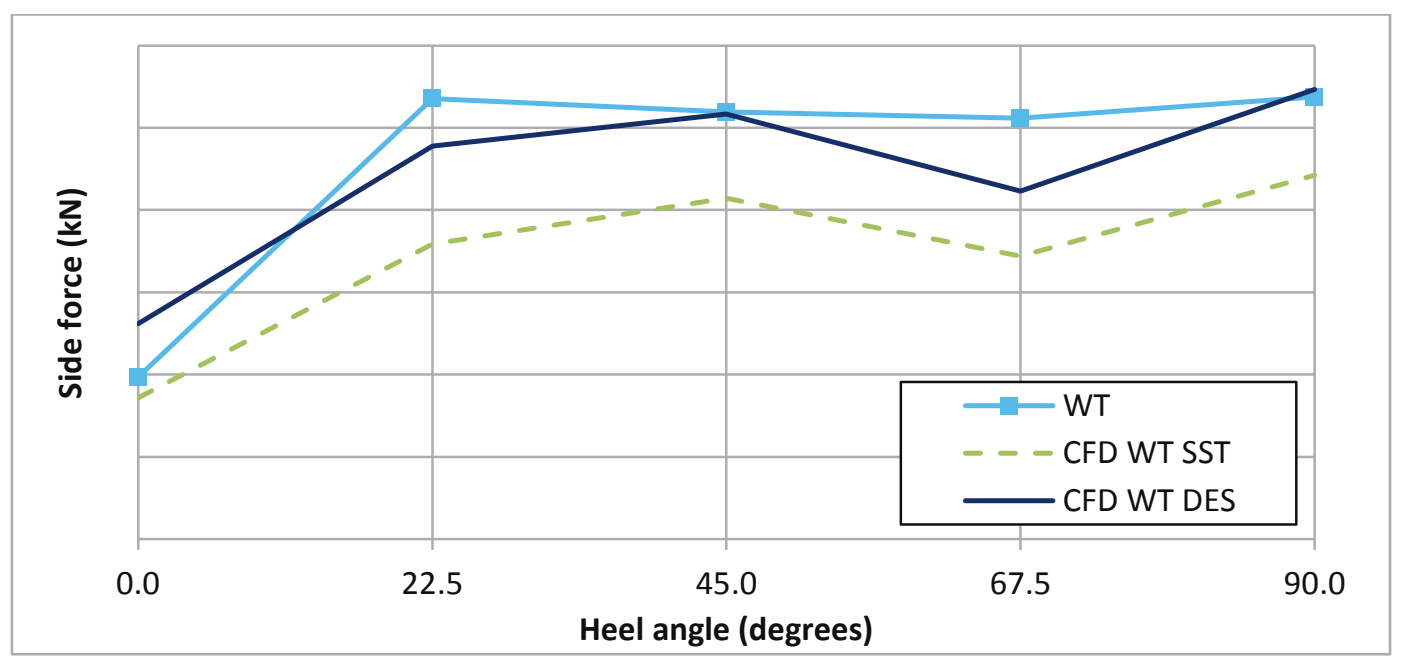

Figure 9: Full scale ACPB side force, WT domain, 40m/s

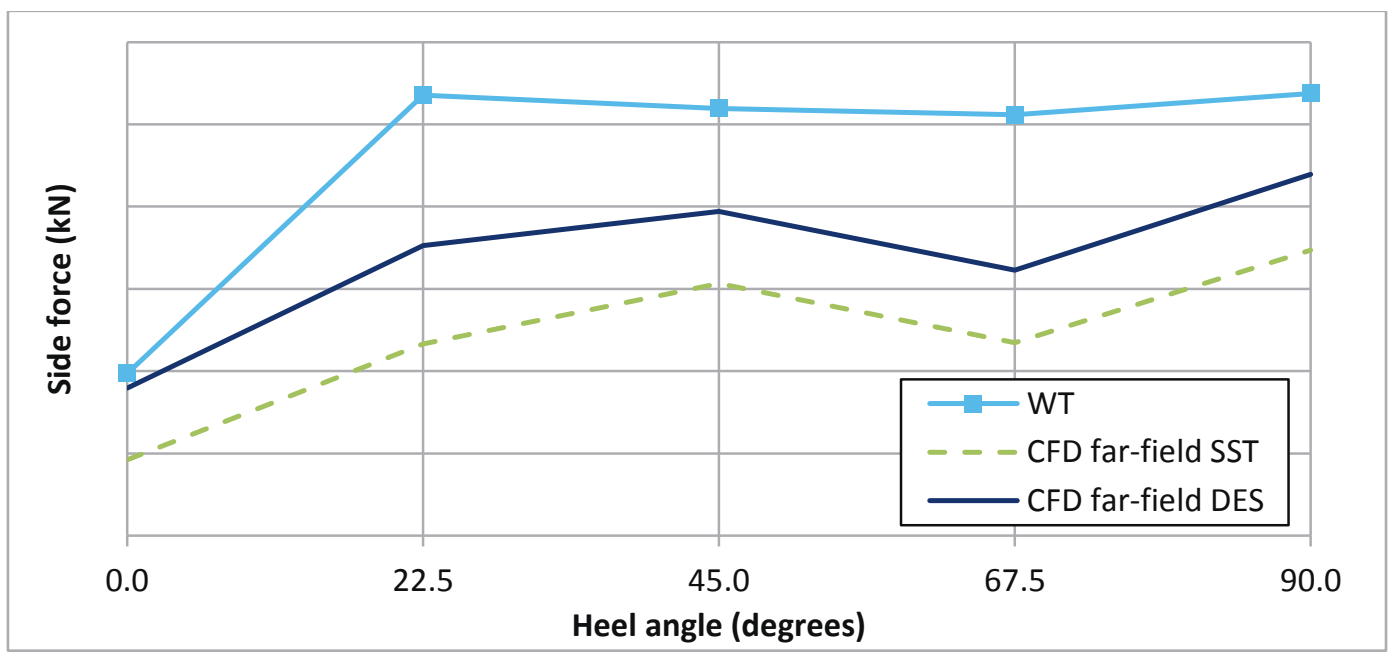

Figure 10: Full scale ACPB side force, far-field domain, 40m/s

A comparison of the two domain types highlight the blockage effect caused by the WT walls, resulting in a drop in side force, whilst the offset between SST and DES in both domains remains similar.

The differences between turbulence models show a very similar trend to the model scale results, where it was shown to favour the DES model due to its ability to more-accurately capture the turbulent wake directly to leeward of the superstructure.

The variation in how the domain is modelled is shown to have a significant influence on the result, of similar magnitude to the variation in turbulence model. This is due to the WT domain constraining the flow at the walls and the ACPB geometry causing a significant blockage, constricting the flow through the WT section, thus increasing the flow and increasing the side force. This constriction and increase in flow is not seen with the farfield domain as the boundaries are both open and of significant distance from the ACPB geometry.

The CFD WT DES results compared to the scaled up WT results show that the model scale CFD methodology is applicable at full scale; however the intention is to predict wind heeling forces and moments at full scale in open water conditions, therefore the far-field domain is the most suitable domain to use.

By assessing the two turbulence models at full scale using the two CFD domains, it was possible to conclude that the use of the DES turbulence model was applicable at full scale. Drawing from these conclusions, the DES turbulence model was used in combination with the far-field domain in order to determine the full scale wind heeling forces and moments acting upon the ACPB at 40m/s wind speed. 


\subsection{CFD full scale heeling moment compared to stability criteria}

DefStan 02-900 [1] states that in current naval stability criteria, the wind heeling moment is calculated for an upright intact ship, and is then assumed to reduce with angle of heel as a function of $\cos ^{2} \varnothing$. This is shown in equation 1 , where $\mathrm{M}_{\mathrm{W}}(\varnothing)$ is the wind heeling moment for a given heel angle, $\varnothing$, and $\mathrm{M}_{\mathrm{W}}(0)$ is the wind heeling moment upright, above the waterline.

$$
\mathrm{M}_{\mathrm{W}}(\varnothing)=\mathrm{M}_{\mathrm{W}}(0) \cos ^{2} \emptyset
$$

According to Brown [5], Japanese experimenters [6] have proposed equation 2 as it has shown to be a better fit to model tests, effectively reducing the heeling moment as a function of $\cos ^{3} \varnothing$.

$$
\mathrm{M}_{\mathrm{W}}(\varnothing)=\mathrm{M}_{\mathrm{W}}(0)\left[0.25+0.75 \cos ^{3} \varnothing\right]
$$

$\mathrm{M}_{\mathrm{W}}(0)$ for both equations can be found empirically using equation 3 [7], where $\mathrm{A}$ is the projected profile area of the ship, above the waterline, $\mathrm{V}$ is the nominal wind velocity and $\mathrm{L}$ is the lever arm from half draught to the centroid of the projected area.

$$
\mathrm{M}_{\mathrm{W}}(0)=0.17 \mathrm{~A} \mathrm{~V} \mathrm{~V}^{2} \mathrm{~L}
$$

A more accurate way of finding $\mathrm{M}_{\mathrm{W}}(0)$ empirically is to divide the profile area into a number of horizontal strips of constant height. A wind profile can then be used where nominal velocity is replaced by the wind velocity at a corresponding strips centroid height. Finally, $\mathrm{L}$ is replaced by the lever arm from half draught to the centroid of each strip, so as to carry out a vertical integration [7]. Alternatively, $\mathrm{M}_{\mathrm{W}}(0)$ can be found from CFD or WT measurement.

The $\cos ^{2} \varnothing$ function assumes that the wind moment decreases proportionally to $\cos ^{2} \varnothing$ as heel angle increases, so that at a heel angle of 90 degrees, the wind heeling moment is zero. The $\cos ^{3} \varnothing$ function assumes a percentage of the wind moment is still acting upon the ship at 90 degrees of heel.

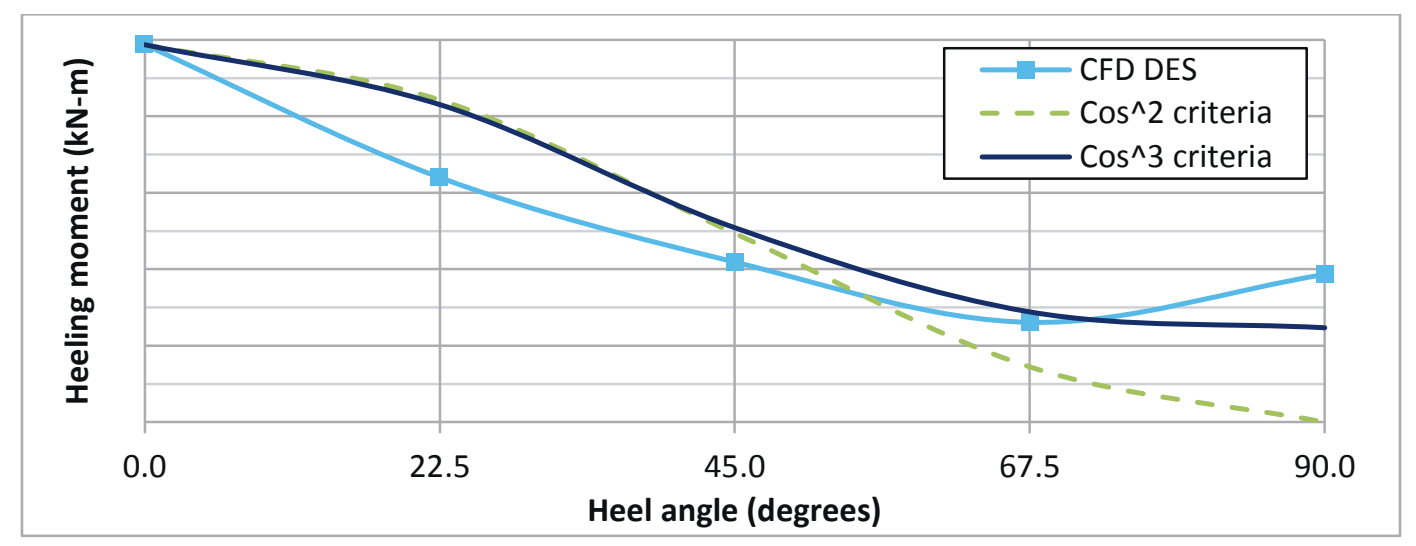

Figure 11: Full scale ACPB heeling moment comparison with stability criteria

Figure 11 plots the heeling moment for the CFD simulations using the far-field domain and DES turbulence model. Plotted alongside the CFD heeling moment (CFD DES) are the two above-mentioned wind heeling criteria; the $\cos ^{2} \varnothing$ criteria $\left(\operatorname{Cos}^{\wedge} 2\right.$ criteria) and the $\cos ^{3} \varnothing$ criteria $\left(\operatorname{Cos}^{\wedge} 3\right.$ criteria).

All three sets of results begin at $\mathrm{M}_{\mathrm{W}}(0)$, which is the wind heeling moment predicted by the CFD simulation in the upright condition.

Comparing the CFD heeling moments with those using the two wind heeling moment functions, it could be concluded that both functions over predict compared to CFD for angles of heel up to 45 degrees. Beyond 45 degrees the $\cos ^{2} \varnothing$ function heavily under predicts heeling moment as it reduces to zero at 90 degrees, whereas the $\cos ^{3} \varnothing$ function is closer to the CFD heeling moments as it reduces to $25 \%$ of MW(0) at 90 degrees.

The use of either function would result in a fair approximation of the wind heeling moment with increased heel angle, as the heeling moment results from CFD were shown to reduce at a similar rate, for angles up to 67.5 degrees. The fact that both functions over predict wind heeling moment up to 45 degrees of heel could be considered a factor of safety, in the absence of full CFD data. 


\section{Full scale predictions of a modern frigate}

Following successful benchmarking of the ACPB geometry at both WT and full scale, decisions were made to improve the CFD process, with the aim of making the process more relatable to an open sea condition. A literature review of articles relating to the implementation of an Atmospheric Boundary Layer (ABL), and the use of DES to resolve small scale turbulence was carried out in support of the decisions made. The resulting improvements to the process were then applied to a full scale analysis of a modern frigate geometry. The ship was artificially heeled at 10 degree intervals and subjected to a 90 knot beam wind.

\subsection{Implementation of an $A B L$}

In a paper by Forrest and Owen [8], a full scale at-sea test is detailed of a frigate airwake performed by DRA (now QinetiQ). It was noted that because the data was obtained at sea, it was necessary to model the effects of the earth's ABL in the CFD airwake computations carried out. In the paper the following power law (4) was used in combination with a zero shear-stress wall to reduce the velocity near the water surface:

$$
\mathrm{U}=\mathrm{U}_{\mathrm{ref}}\left(\frac{\mathrm{Z}}{\mathrm{Z}_{\mathrm{ref}}}\right)^{\propto}
$$

Where $\mathrm{U}_{\text {ref }}$ is the velocity at the reference height, $\mathrm{Z}_{\text {ref }}$, and $\alpha$ is a constant dependant on surface terrain. They concluded that the implementation of an ABL in the CFD computations showed better agreement with full scale experimental data.

DefStan 02-900 [1] states that for stability in beam winds, the wind heeling levers are obtained using the wind velocity profile defined in equation 5 :

$$
\mathrm{U}=\mathrm{U}_{\mathrm{ref}}\left(\frac{\mathrm{Z}}{10}\right)^{1 / 7}
$$

The way of defining the ABL in DefStan is very similar to that prescribed by Forrest and Owen. As comparisons would be made between CFD wind heeling calculations and the wind heeling criteria defined in DefStan, equation 5 would be used, but in combination with a zero shear-stress wall as recommended by Forrest and Owen.

\subsection{Unsteady time-step and resolution of small scale turbulence}

During the CFD benchmarking process, the time-step, $\Delta_{T}$, was normalised by $\mathrm{U}_{\text {ref }}$, and ship length, L, with the aim to have one hundred steps over the length (6):

$$
\Delta_{\mathrm{T}}=0.01 \frac{\mathrm{L}}{\mathrm{U}_{\mathrm{ref}}}
$$

This assumption was valid for the WT analysis of the ACPB, where good agreement between CFD DES computations and the raw wind tunnel data was observed. The modern frigate had a greater length to beam ratio, so in order to maintain a courant number of $<1$, the $\Delta_{T}$ was normalised using the ship's beam, B, which is consistent with Forrest and Owen [8], where a frigate airwake was modelled in a headwind, using the K-Omega DES turbulence model with similar $\Delta_{\mathrm{T}}$, based on guidelines from [9, 10 \& 11]. 


\subsection{Changes to the heeling lever centre}

Up until this point the assumption has been that the ship has been heeled about the keel line, as it was in the WT experiment. This was a valid assumption when comparing to WT measurements, however a more accurate representation of the heeling moment acting on the ship should depend on the axis in which the ship would heel; the ship's Centre of Gravity (COG). The methodology was therefore altered such that the ship was artificially heeled about the COG rather than about the keel line, still maintaining a constant displaced volume. Wind heeling moments were then taken about the COG.

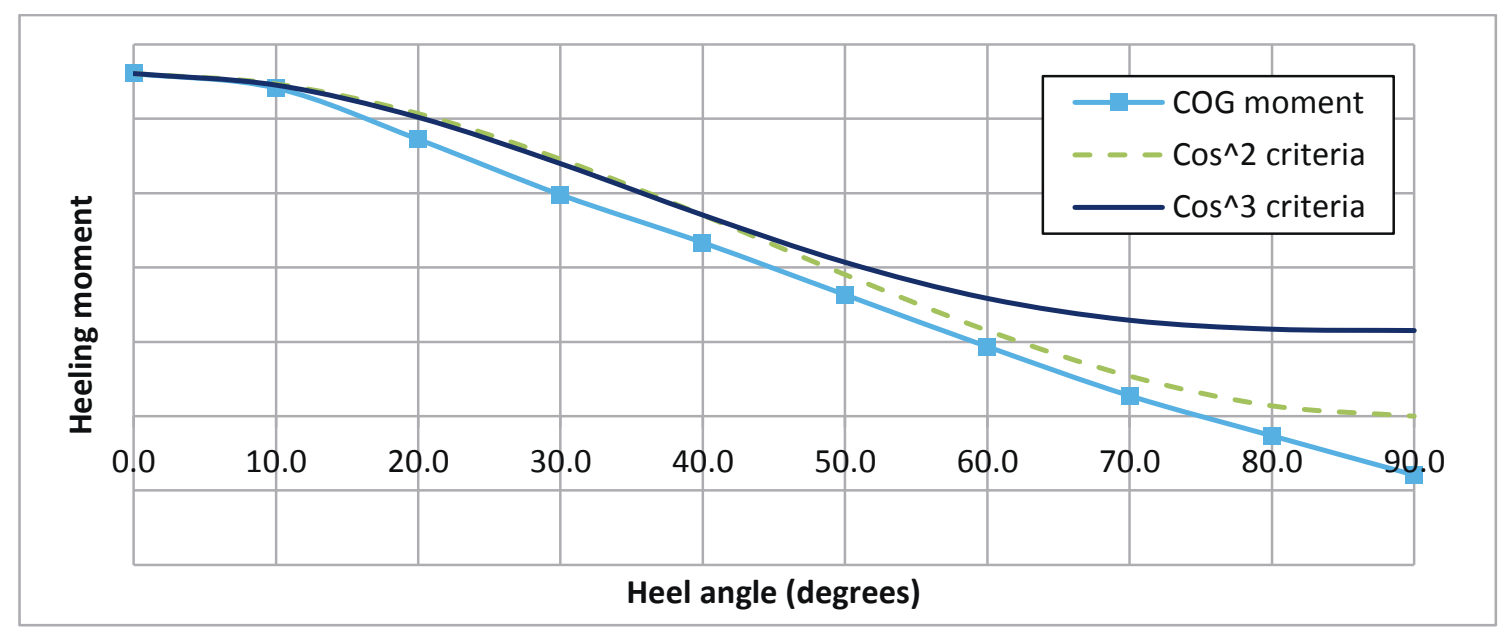

Figure 12: Modern frigate heeling moment vs heel angle

Figure 12 plots heeling moment against heel angle, along with the two above-mentioned stability criteria; $\cos ^{2} \varnothing$ and $\cos ^{3} \varnothing$, with $\mathrm{M}_{\mathrm{W}}(0)$ dependant on the upright COG moment.

When the criteria are dependent on $\mathrm{M}_{\mathrm{W}}(0)$, taken about $\mathrm{COG}$, both match the CFD results well, up to 60 degrees of heel.

DefStan 02-900 states that the angle of inclination due to beam winds must not exceed 30 degrees of heel when subjected to the nominal wind speed. The nominal wind speed is dependent on the type of ship, so for an ocean going ship of monohull form, the nominal wind speed would be 90 knots. Based on this assumption, there is a reason to limit the angle of heel to be compared with stability criteria.

\subsection{Modern frigate vector flow field}

Figure 13 shows mean velocity vectors of the wake on a plane passing through the centre of the modern frigate mast. The turbulent eddies within the wake are clear, showing recirculation impacting on the leeward side of the ship. The stagnation point on the windward side of the hull is also clear to see, as is the separation off the bilge keels at high angles of inclination.

Based on the recommendation of Forrest and Owen [8] to model the sea surface as a zero-shear stress wall, the ABL was expected to be maintained, yet this is not the case. Due to the limitations imposed by the boundary conditions, the ship will not see the profile applied at the inlet, be it due to the zero-shear wall or the turbulence of a non-slip wall applied in the WT CFD methodology. 


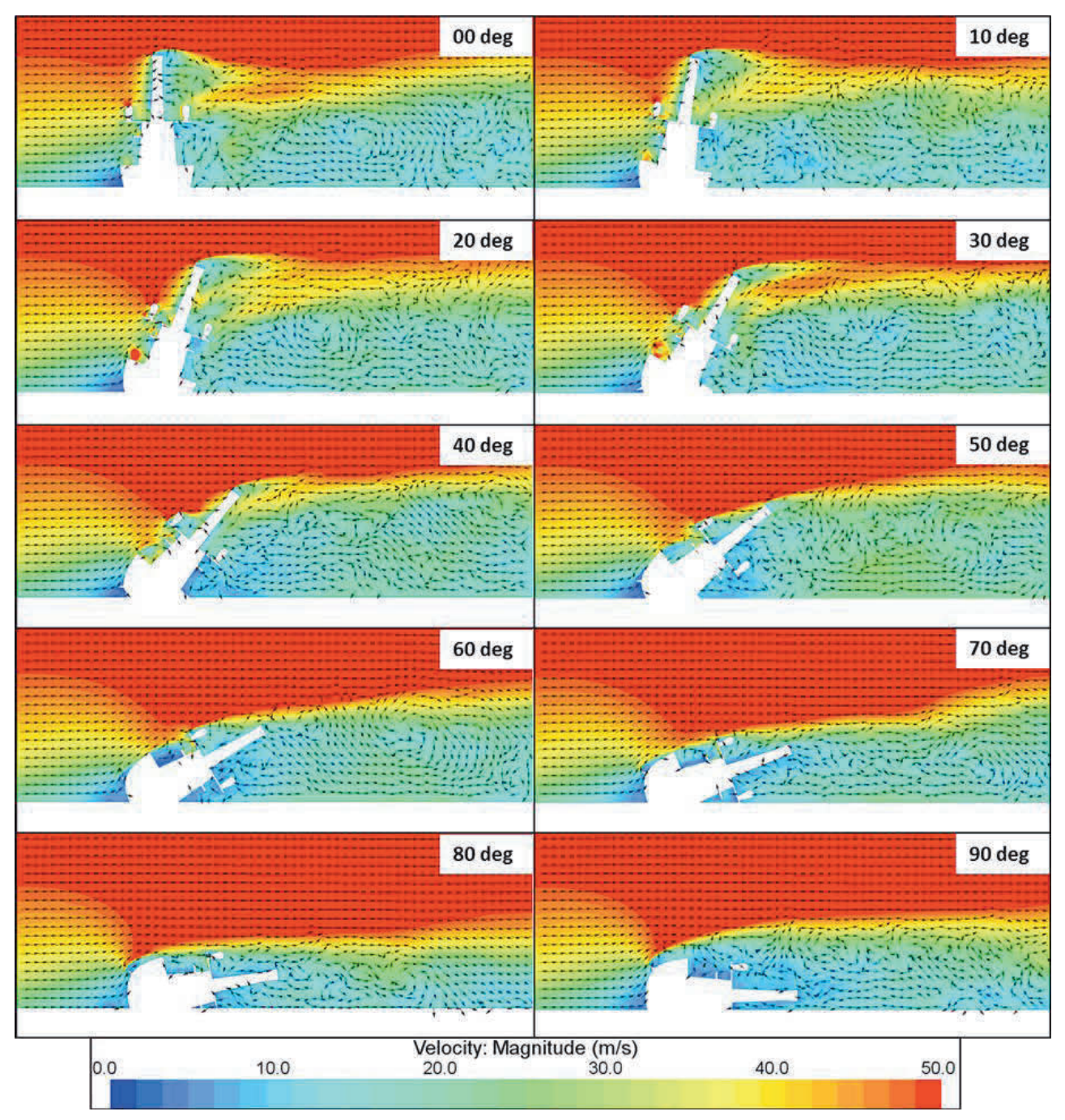

Figure 13: Modern frigate mean velocity vectors

\section{Concluding remarks}

The use of WT experimental data to benchmark a CFD process has proven to be successful, predicting the wind heeling moment using CFD to within $4 \%$ of the WT result when using the DES turbulence model. When comparing full scale CFD with scaled up WT data, the results correlated well when using a scaled up WT CFD domain. Based on this correlation, it was concluded that the model scale CFD procedure was applicable at full scale using the DES turbulence model.

The modern frigate results have shown that the use of the COG as the axis for heel is a more accurate representation of a ship subjected to a beam wind.

Using COG in the calculation of $\mathrm{M}_{\mathrm{W}}(\varnothing)$ has shown that either criterion could be considered acceptable up to 40 degrees; 10 degrees beyond the DefStan exceedance angle, and that based on the $\mathrm{CFD}_{\mathrm{W}}(\varnothing)$ calculations, the criterion could be considered to include a margin of safety due to their slight over-prediction.

\section{Recommendations \& further work}

The addition of an ABL has made the CFD process more representative of the real world. However, there are limitations imposed by the boundary conditions which impact the wind profile seen by the ship. It is recommended that further work be carried out to define boundary conditions which are more representative of the sea surface and its boundary layer, and in doing so maintaining the profile imposed at the inlet, for example the inclusion of surface roughness. 


\section{References}

[1] UK Defence Standardization: "Stability Standards for Surface Ships (Cat 1)", Part No: 1 - Conventional Ships, Def Stan 02-900, May 2014.

[2] Sarchin T.H. \& Goldberg L.L.: "Stability and buoyancy Criteria for U.S. Naval Surface Ships", S.N.A.M.E., 1962.

[3] Khee C.H.: "Variation of wind heeling moment with angle of heel of ships", University of New South Wales, Australia, 3229936, October 2010.

[4] Edwards C.L.: "Ship Wind Heeling Measurement and Comparison with Numerical Analysis", University of New South Wales, Australia, z3254386, June 2015.

[5] Brown P.: “A Study of Small Ship Stability”, Department of Defence, Canberra.

[6] Kinoshita M. \& Okada S.: "Heeling Moments due to the Wind Pressure on Small Vessels", Symp. On the behaviour of ships in a seaway, Wageningen, Netherlands, P.527, 1957.

[7] Rawson K.J. \& Tupper E.C.: "Basic Ship Theory", Volume 1, Chapters 1 to 9, Hydrostatics and Strength, Longman Scientific \& Technical, Fourth edition, 1994.

[8] Forrest J.S \& Owen I.: “An investigation of ship airwakes using Detached-Eddy Simulation”, University of Liverpool, Computers and Fluids 39 656-673, November 2009.

[9] Spalart P.R.: "Young-person's guide to detached-Eddy simulation grids", Technical Report, NASA, NASA/CR2001-211032, 2001.

[10] Hedges L.S, Travin A.K, Spalart P.R.: "Detached-eddy simulations over a simplified landing gear", J Fluids Eng, Trans ASME 2002;124(2):193-200, 2002.

[11] Forsythe J.R, Squires K.D, Wurtzler K.E, Spalart P.R.: "Detached-eddy simulation of the F-15E at high alpha”, J Aircraft 2004;41(2):193-200, 2004. 